\title{
Lupus anticoagulant in children - a confounding factor in diagnosis and targeted therapy
}

\author{
Cristian Jinca ${ }^{1 *}$, Margit Serban ${ }^{2}$, Emilia Ursu $^{3}$, Nicoleta Anamaria Pascalau ${ }^{4}$, \\ Oana Belei ${ }^{1}$, Delia Savescu ${ }^{5}$, Mihaela Lelik ${ }^{5}$, Andrei Ioan Munteanu ${ }^{6}$, Andreas \\ Tiede $^{7}$, Smaranda Arghirescu ${ }^{1}$
}

1. Department of Pediatrics, "Victor Babeș" University of Medicine and Pharmacy Timişoara, Romania

2. Department of Onco-hematology, Romanian Academy of Medical Sciences, Onco-Hematology Research Unit, Children Emergency Hospital “Louis Turcanu” Timisoara, European Hemophilia Treatment Centre, 300011 Timisoara, Romania

3. Department of Onco-hematology, Onco-Hematology Research Unit, Children Emergency Hospital

“Louis Turcanu” Timisoara, European Hemophilia Treatment Centre, 300011 Timisoara, Romania

4. Department of Psycho-neurosciences and Rehabilitation, University of Oradea, Faculty of Medicine and Pharmacy, Romania

5. Department of Clinical Laboratory, Children Emergency Hospital “Louis Turcanu” Timisoara, 300011 Timisoara, Romania

6. Department of Puericulture and Neonatology, "Victor Babeș" University of Medicine and Pharmacy Timişoara, Romania

7. Clinic for Hematology, Hemostasis, Oncology and Stem Cell Transplantation, Hannover Medical School, Germany

\begin{abstract}
Introduction: Lupus anticoagulant (LAC) belongs to a heterogeneous group of antibodies directed against negatively charged phospholipid-binding proteins, inhibiting phospholipid-dependent reactions. We assessed the frequency, etiological background, clinical and biological expression as well as the appropriate management and outcome of LAC in a pediatric population. Patients and methods: We included 19 pediatric patients diagnosed with
\end{abstract}

* Corresponding author: Cristian Jinca, Department of Pediatrics, "Victor Babeș" University of Medicine and Pharmacy, Timisoara, Romania. E-mail: cristian_jinca@yahoo.com 
a positive LAC test during a period of 1 year. LAC detection was carried out according to the ISTH/SCC guidelines 2012. Coagulometric assays were performed in order to check for coagulation factor activities. The presence of specific inhibitors has been tested by the Bethesda assay. Results: LAC was positive in $0.4 \%$ of the patients monitored for increased activated partial thromboplastin time (aPTT) during the study period of 1 year. In contrast to the asymptomatic clinical image presented by the majority of our patients, the biological picture was dominated by an elevated aPTT (79\%), the aPTT ratio correlating significantly with the strength of LAC. In $58 \%$ of the patients LAC became negative within 12 weeks, in another 4 (21\%) patients within 20 weeks, whereas in 4 (21\%) patients $L A C$ remained positive throughout the follow-up. Conclusion: Albeit usually innocent in asymptomatic patients, $L A C$ needs to be seriously taken into consideration from the diagnostic point of view in order to avoid dangerous therapeutic attitudes..

Keywords: antiphospholipid syndrome, acquired hemophilia, activated partial thromboplastin time, lupus anticoagulant

Received: 19 $9^{\text {th }}$ March 2021; Accepted: $21^{\text {st }}$ June 2021; Published: $10^{\text {th }}$ July 2021

\section{Introduction}

Lupus anticoagulant (LAC) is considered a well-established, very sensitive risk factor and main predictor for thrombotic events in adults. It belongs to the heterogeneous group of antiphospholipid antibodies, directed against negatively charged phospholipid-binding proteins, thereby inhibiting phospholipid-dependent reactions (1). Its identification relies on a combination of two phospholipid (PL)-dependent functional clotting assays, as no single test has sufficient sensitivity and specificity for the diagnosis (2). Even in this case, these assays are not robust enough, lacking accuracy as do one stage coagulometric assays, thus requiring sometimes access to radioimmunoassay, ELISA or solid phase antigen tests. LAC is a true double misnomer, not being a specific marker for systemic lupus erythematosus (SLE) and in fact not being a real promoter of anticoagulation. On the other hand, LAC may be at the same time a biological marker for the antiphospholipid syndrome (APS), even in a catastrophic form, as it can also be a simple, innocent bystander, a laboratory phenomenon without any clinical consequences.
In children LAC is often found incidentally, frequently assessed in the frame of investigations for a prolonged activated partial thromboplastin time (aPTT) prior to surgery or during/after viral infections, usually disappearing within 12 weeks $(3,4)$. It is an exceptionally rare cause of bleeding disorders as it would be in the case of the lupus anticoagulant hypoprothrombinemia sydrome (LAHS) which is sometimes associated with thrombocytopenia and is also seldom the isolated substrate for thrombotic events $(1,4,5)$. Inhibiting phospholipid-dependent reactions, LAC can have confounding effects in the diagnostic field of hemostasis by mimicking haemophilia and leading to the misdiagnosis of acquired haemophilia, thus impacting the therapeutic decision, engaging high costs for exploration and sometimes even unnecessary treatments associated with important side effects. Our objectives were to conduct an observational, populational and non-interventional single center study, aiming to assess the frequency, the etiological background, the clinical and biological expression as well as the outcome of LAC in a pediatric population and to evaluate the most appropriate attitude in front of it. 


\section{Material and methods}

Out of 4,607 patients consecutively explored with basic hemostasis tests form a total of 17,800 patients consecutively admitted during a period of 1 year, we retrospectively enrolled in our study 19 patients with a positive LAC test. The LAC test was performed for: a) unexplained, repeatedly elevated aPTT and aPTT ratio in asymptomatic patients with normal prothrombin time (PT), thrombin time (TT), fibrinogen and prothrombin consumption test (PCT), lacking correction by the mixing test with normal pooled plasma; b) autoimmune diseases and c) thromboembolic disorders.

For the aPTT screening we used the APTT-SP (liquid) kit. A contact activator is used to stimulate the production of factor XIIa by providing a surface for the function of high molecular weight kininogen, kallikrein and factor XIIa. This contact activation is allowed to proceed at $37^{\circ} \mathrm{C}$ for a specific period of time. Calcium is then added to trigger further reactions and the time required for clot formation is measured. Phospholipids are required to form complexes which activate factor $\mathrm{X}$ and prothrombin. The kit contains synthetic phospholipids and silica to ensure a highly reproducible and stable product. Reference range for aPTT was (23-36 seconds). The aPTT ratio (patient aPTT/mean normal aPTT) was calculated considering the dependence on the used reagent and accepting as normal values 0.82 1.2. The mixing study was performed measuring aPTT in 1:1 mixture of patient plasma and normal pooled plasma. Testing for LAC was done if the addition of normal pooled plasma to the patient's plasma did not correct the aPTT; although this was not an inclusion criterion, most patients had a second LAC test within 4 or 8 weeks but all patients were checked at 12 and and also beyond 12 weeks after the initial test for the patients who continued to present positive LAC. For LAC detection, the screening and confirmatory tests
(Hemosil LAC Confirm, Bedford, USA) based on dilute Russel's viper venom time (dRVVT) were used. The dRVVT Screen assay is poor in phospholipid, making it sensitive to LAC. The additional amount of phospholipid (bi-layers) in the dRVVT Confirm assay neutralizes LAC to give shorter dotting times. Russell's Viper Venom, in the presence of calcium, directly activates factor X (in the test sample). The dRVVT screen and dRVVT Confirm assays are therefore unaffected by contact factor abnormalities, coagulation factor VII (FVII), VIII (FVIII) and IX (FIX) deficiencies or inhibitors. Heparin interference up to $1 \mathrm{U} / \mathrm{ml}$ is neutralized by polybrene.

All tests were carried out according to the manufacturer's instructions and to the ISTH/SCC guidelines 2012 with a three-step method including a mixing test (2). They were performed on the ACL TOP automated coagulation analyser (Instrumentation Laboratory, Bedford, USA). LAC was considered positive if $>1.2$. The diagnostic work-up included in most cases an extensive hemostasis exploration: PT (reference range 9-14 seconds), international normalized ratio (INR) with the reference range between $0.8-1.2$, TT (reference range 12-20 seconds), fibrinogen levels (reference range 200-400 mg/ $\mathrm{dL}$ ), PCT (reference range $>40$ ). At the same time, only in primary LAC patients (74\%) coagulometric assays were carried out for the level of FVIII, FIX and FXI activity measurement (table 1). FVIII, FIX and FXI activity was determined by performing a modified activated partial tromboplastin time test (APTT-SP). Patient plasma is diluted and added to plasma deficient in factor VIII/IX/XI. Correction of the clotting time of the deficient plasma is proportional to the concentration ( $\%$ activity) of the factor in the patient plasma, interpolated from a calibration curve.

For patients with low levels of coagulation factors, the presence of specific inhibitors has been tested by means of the Bethesda assay and in the case of a positive result a confirmation testing by 
ELISA (ZYMUTEST ${ }^{\mathrm{TM}}$, Hyphen BioMed) has been done. In patients with increased aPTT ratio and low coagulation factors activity we performed also thrombelastography (TEG).

For the statistical analysis of the parametric correlations, the IBM ${ }^{\circledR}$ SPSS ${ }^{\circ}$ Statistics software version 25 was used. The cut-off level for significance was $p<0.05$. Patient's or caregiver's consents were obtained. All procedures were performed in accordance with the standards of the institutional ethics committee which approved the study.

\section{Results}

Mean age at diagnosis was $6.15 \pm 5.09$ years, with a median of 4.20 years (range between 1.5 -18 years) and a male/female ratio of 7/12. All patients had newly detected LAC (patients with known and confirmed positive LAC were excluded from the analysis) out of 4607 patients consecutively explored with basic hemostasis tests from a total of 17,800 patients admitted in our hospital during a period of 1 year. The positivity of the LAC test was ascertained in $0.4 \%$ of our paediatric cohort of children found to have a prolonged aPTT, coresponding to a frequency of $10.7 / 10,000$ hospitalized persons and year. Our cohort had in 12 cases elevated aPTT incidentally found during pre-operative work up whereas in 3 cases immune vascular or thrombocytopenic purpura (1 immune thrombocytopenic purpura ITP, in which the acquired LAHS and APS were excluded, 1 Hennoch-Schonlein purpura - HSP and 1 Seidelmeyer purpura - SP), in 2 cases systemic lupus erythematosus (SLE) and in 2 other cases an unprovoked thrombotic event (APS). Nine $(75 \%)$ of the 12 patients with incidentally found LAC could recall having a upper respiratory tract infection in the weeks prior to the test. Based upon its strength, LAC was weakly present $(1.2-1.5)$ in 10 patients, moderately $(1.5$ $-2.0)$ in 4 and strongly present $(>2.0)$ in 5 patients (figure 1). In 2 patients LAC was considered secondary to SLE, in other 3 to immune or vascular purpura whereas the rest of 14 cases were considered as having primary LAC.

At the time of the first detection LAC was asymptomatic in the majority $(63 \%)$ of our patients. Only 3 patients $(15.8 \%)$ presented mild

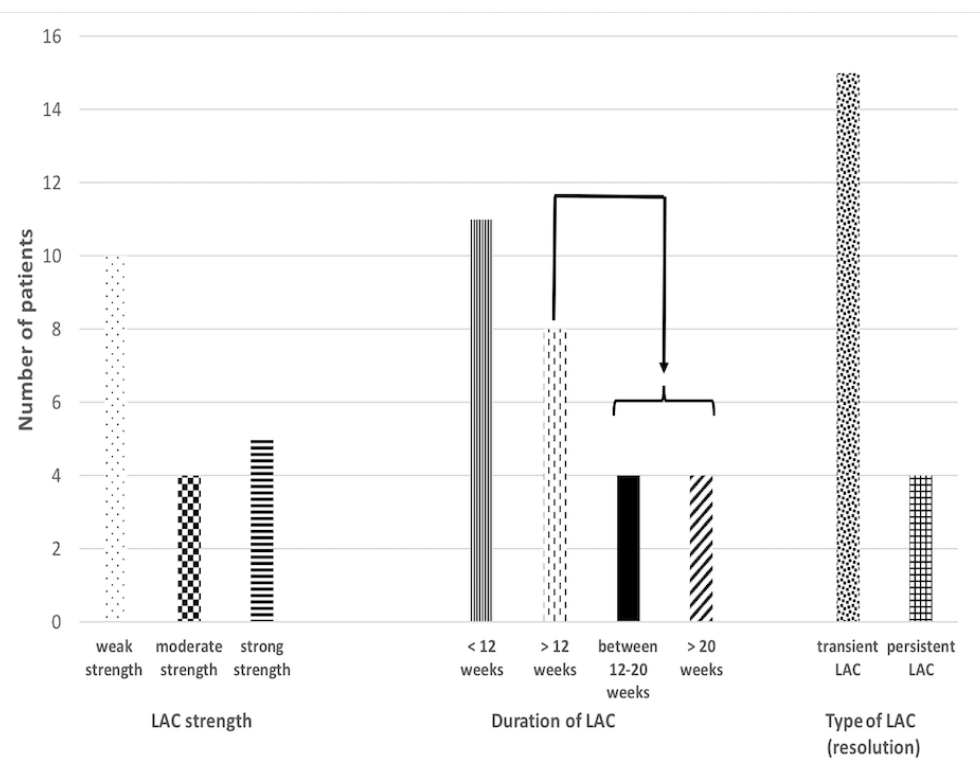

Fig. 1. Distribution of LAC according to its strenght and time to resolution 
cutaneous bleeding, petechiae and/or equimoses, attributable to their causative pathologic background (ITP, HSP, SP). Two patients had thrombosis and other 2 patients SLE (table 1).

The biological picture was dominated by an elevated aPTT, present in 15 cases (79\%), only the two patients with SLE and the two patients with APS having normal aPTT. All of the patients with elevated aPTT presented with an aPTT ratio of $>1.3$, the mean aPTT ratio being $2.34 \pm 1,13$. The aPTT ratio correlated significantly with the value of LAC, with a Pearson's $\mathrm{r}=0.817$ ( $p=$ $0.000)$ as presented in figure 2 .

Concerning the clinical and biological parameters of the entire study cohort, they are depicted in tabel 1.

Other coagulation tests including PT, INR, TT and PCT were within the normal range in all subjects (table 1). The coagulation activity of F VIII, IX and XI was assessed in 15 (79\%) of them. Six patients $(32 \%)$ presented the factor levels were lower than $40 \%$; in one case isolated low FIX, in 2 cases low FVIII and FIX, whereas in other
4 cases an association of low FVIII, FIX and FXI levels were found. Nevertheless, from all of these cases which presented reduced FVIII, IX or XI activity, the Bethesda assay was positive for FVIII, FIX and FXI only in 1 patient, showing increasing dinamics and reaching titres of 28.85 Bethesda units (BU)/ml anti-FVIII, 36.9 BU/ml anti-FIX and $5 \mathrm{BU} / \mathrm{ml}$ anti-FXI. In this case, the lack of bleeding symptoms, the normality of the PCT and of TEG doubted the authenticity of the diagnosis of acquired haemophilia $(\mathrm{AH})$, justifying our watch and wait attitude and the need for antigenic testing by means of immunologic assays (ELISA testing) which did not confirm the presence of specific inhibitor antibodies and excluded the presumed complex AH A, B and C. In 8 out of 19 patients in which the aPTT ratio was higher than 2, we also performed TEG. The assays were normal in all cases with the exception of 1 patient (patient no. 1) in which a mild hypercoagulability process was observed, albeit D-dimers were not elevated (figure 3 - TEG parameters: $\mathrm{R}=2.5$, Ly $30=2.3 \%, \mathrm{CI}=4)$.

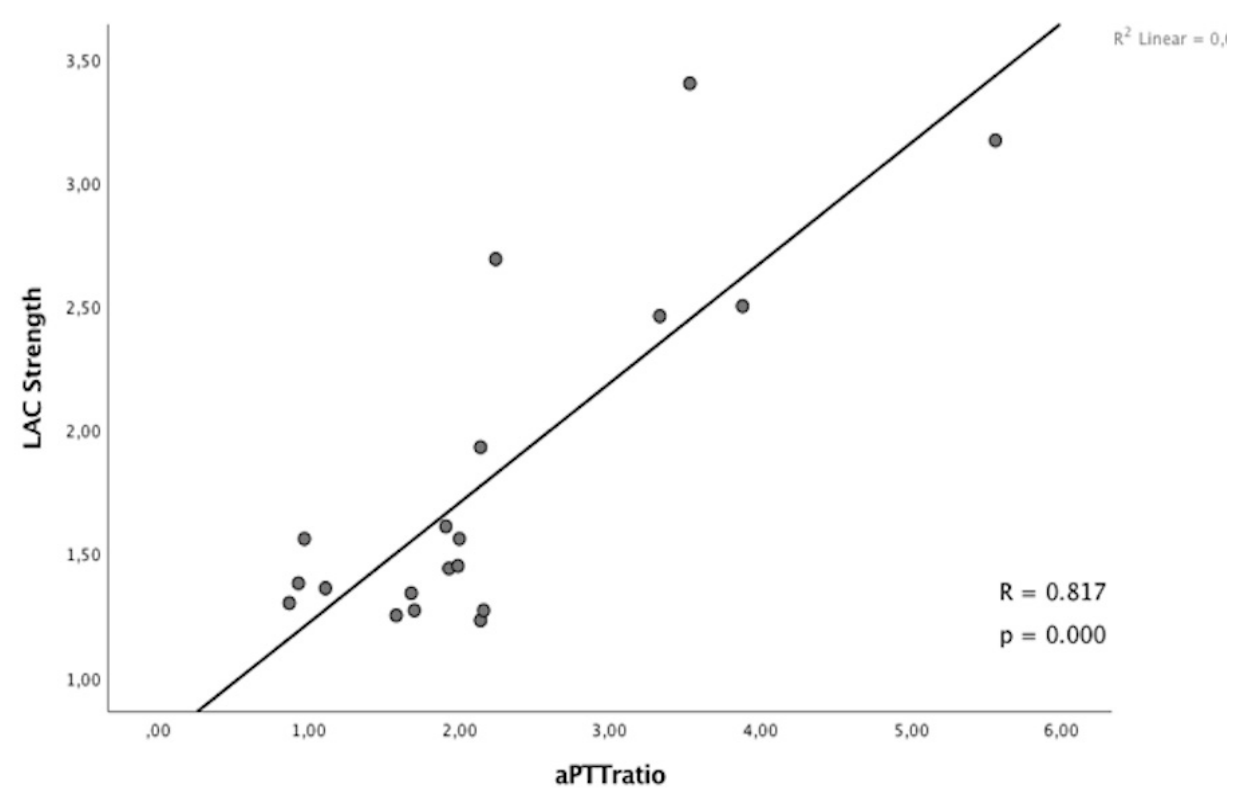

Fig.2. Correlation between aPTT ratio and strength of LAC 


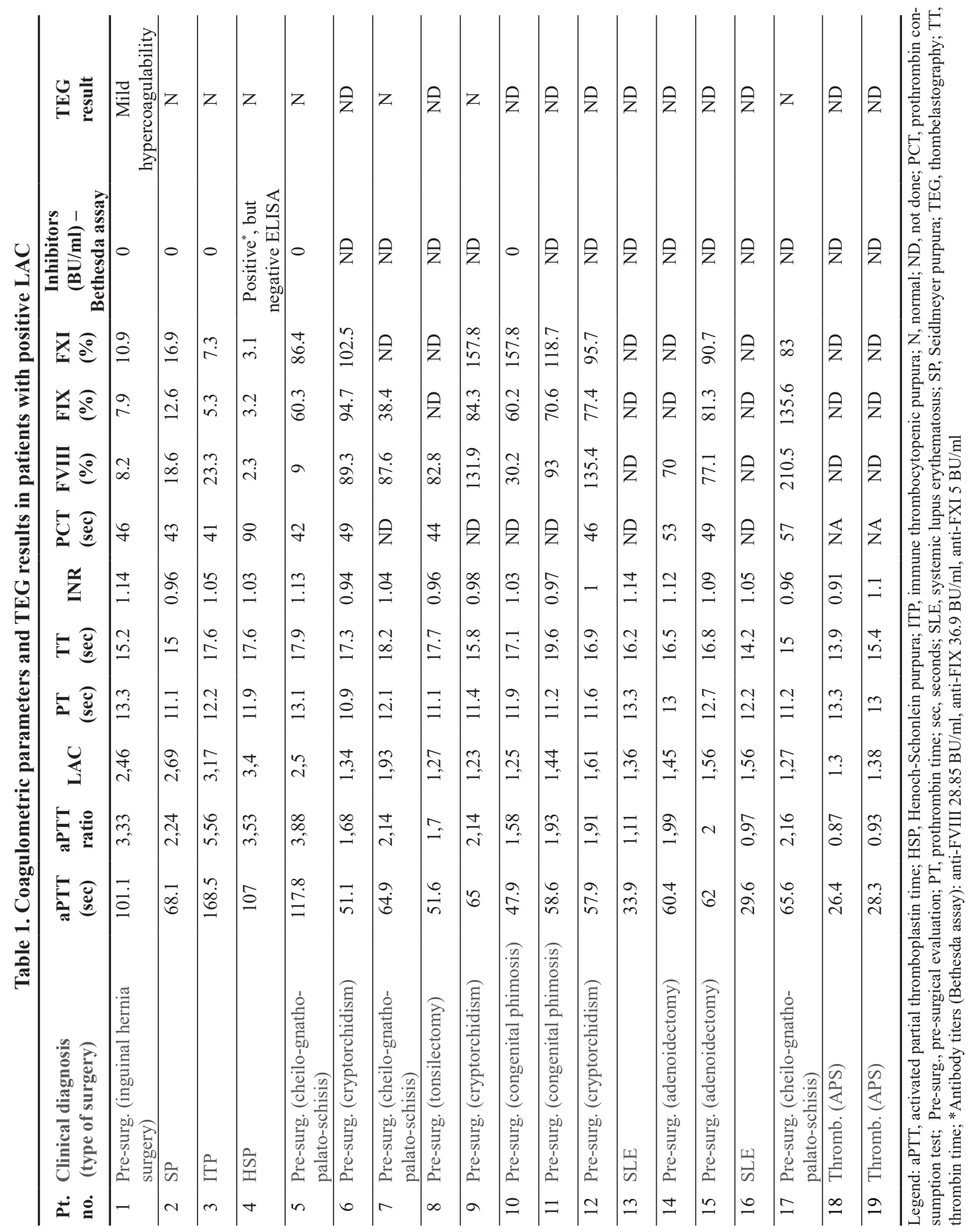




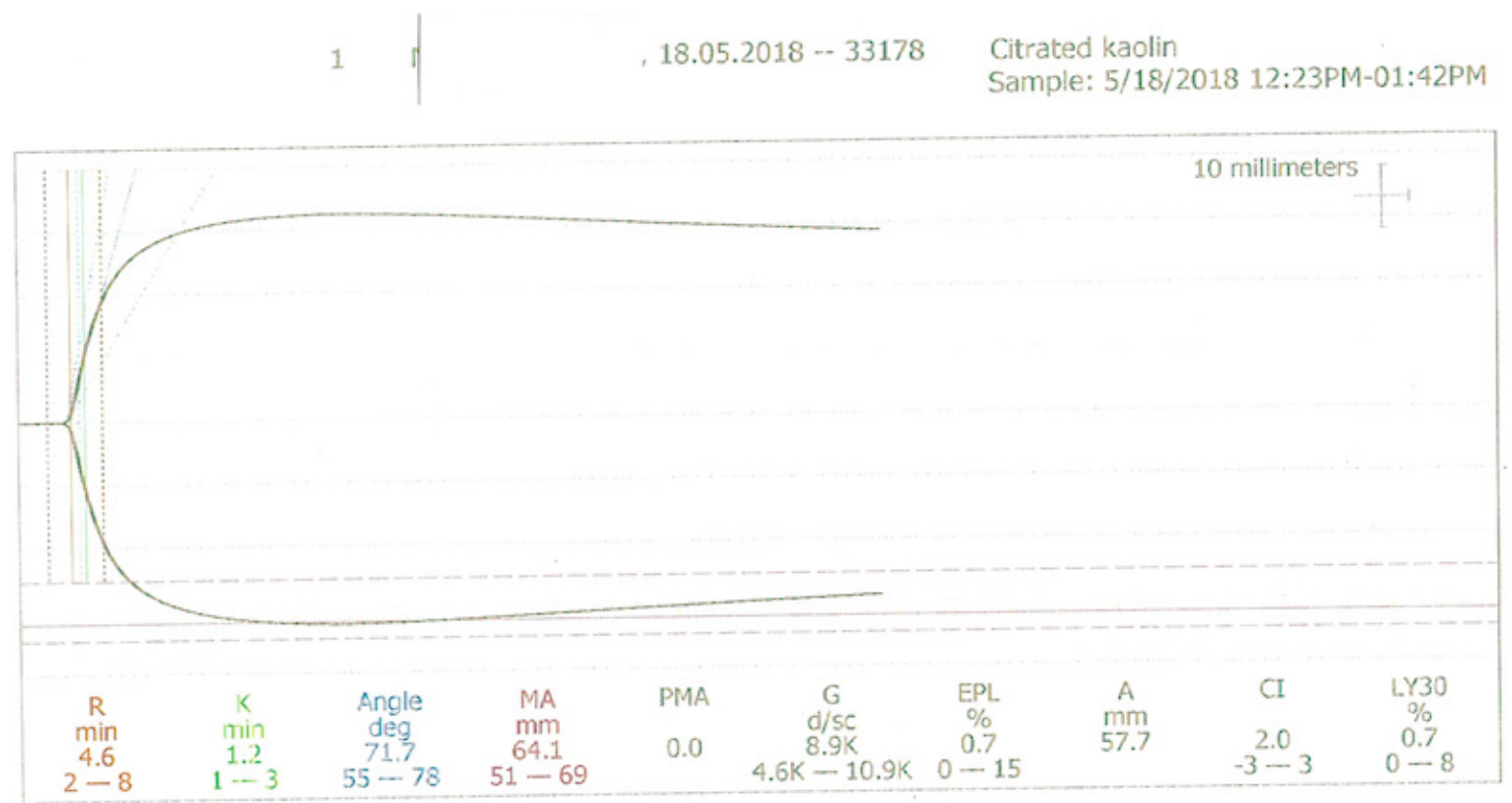

Fig. 3. Thrombelastography showing mild enzymatic hypercoagulability in patient 1

The time course of LAC for the whole cohort is illustrated in figure 1. LAC was transient in 15 patients (79\%), becoming negative within 12 weeks in 11 patients $(58 \%)$ or at the latest within 20 weeks after the initial detection for another 4 patients. Four patients $(21 \%)$, presenting with
APS and SLE, remained positive throughout their follow-up period (at 6, 8, 9 and 10 months check-ups respectively) and thus were considered LAC carriers. The time course of LAC positivity did not correlate with its strength, as shown in figure 4 (Pearson's $\mathrm{r}=-0.272 ; p=0.260$ ).

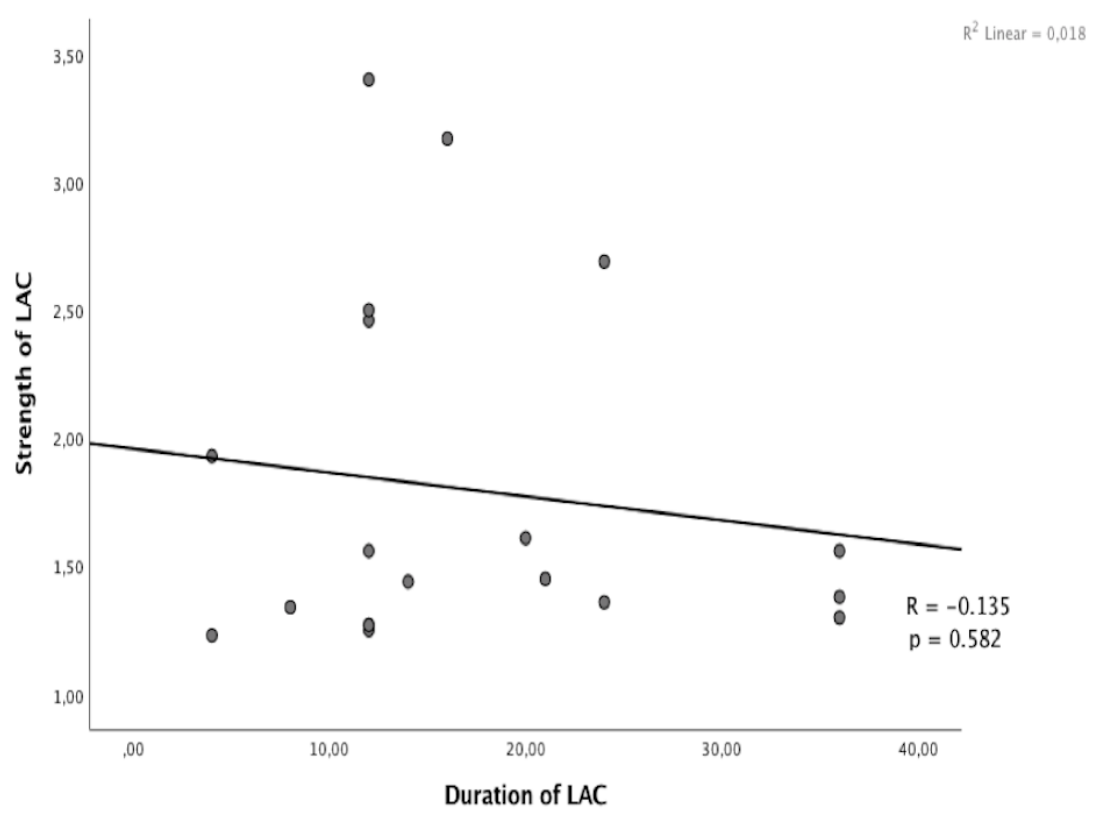

Fig. 4. Scatterplot for correlation assessment between strength and time course of LAC positivity 


\section{Discussions}

LAC, even though rare in children, is nevertheless not an exceptionally occuring disorder $(4,6)$. As reported in the literature, from the point of view of their pathogenic background, LAC in our patients can be considered secondary to immunological disorders (SLE, HSP, SP, ITP) in 5 cases and idiopathic or primary in the other 14 subjects.

With respect to their pathological significance, the majority of our patients presented without symptoms suggestive for a hemorrhagic diathesis or thrombosis; only two patients $(10 \%)$ developed unprovoked thrombotic events, witnessing APS, other three patients presenting minor bleedings, attributable to their underlying diseases (HSP, ITP, SP).

It is generally accepted that the interpretation of an isolated LAC in comparison with its associated form with other antiphospholipid antibodies (aPLs) is a field of many pitfalls. The reason is related to the laboratory assessments. Whilst anticardiolipin (aCL) and anti- $\beta_{2}$ glycoprotein I antibodies ( $\left.\mathrm{a} \beta_{2} \mathrm{GPI}\right)$ are detected by solid phase assays and can be calibrated in order to generate quantitative precise results, LAC are detected by fluid coagulation assays with multifactorial dependence (7-10). Therefore, an isolated form is most often an in vitro phenomenon occuring due to auto-antibodies targeting negatively charged phospholipid-binding proteins, thereby inhibiting phospholipid-dependent coagulation tests such as aPTT. In $63 \%$ of our cases, LAC was from clinical point of view an innocent finding, but it acted in the exploratory field as a serious "troublemaker", generating challenging situations. The outcome towards resolution of LAC within 12 weeks in the majority of our patients $(58 \%)$ as well as the absence of thrombotic or hemorrhagic manifestations during the whole observation period, justified the presumtion that inspite its autoantibody character, LAC was bi- ologically a true confounding factor, impacting only hemostatic explorations, without real clinical relevance.

In this situation, confronted with clinical cases with isolated LAC, sometimes $31.5 \%$ in our group of patients) expressing a challenging biological picture of acquired haemophilia $(\mathrm{AH})$, we raised some questions: which should be the advisable diagnostic and therapeutic approach for these patients in order to avoid unnecessary expensive investigations and treatments, but keeping in mind that $\mathrm{AH}$ must be precisely ruled out; which should be our attitude towards the programmed invasive surgical interventions for patients with pre-surgery detection of isolated elevated aPTT and how should they be monitored. The correctness of some $\mathrm{AH}$ cases with associated LAC reported in the literature have been considered questionable, arguing that the lack of investigation with solid assays rendered the results as false positive $(11,12)$. Taking into account the features of LAC in apparently healthy children, the strong pathogenic connection of LAC with APS, considering also the striking differences between childhood and adult onset APS (13), lacking information on the outcome of persistently positive antibodies, we formulated some topics of interest for pediatricians:

\section{Who should be investigated?}

- symptomatic patients with thromboembolic events and exceptionally with bleeding associated with challenging hemostatic results (13);

- asymptomatic patients with unexplained prolonged aPTT (14);

- newborn babies of mothers diagnosed with APS (15).

\section{Where and when should we perform the explo- ration of $\mathrm{LAC}$ ?}

- in a competent laboratory, with certification for hemostasis investigations or, in cumber- 
some cases, in a reference laboratory with expertise for APS diagnosis (2);

- after repeating the pathological screening aPTT test (2 times during 3-5 days);

- after excluding pre-analytical factors generating artefactually increased aPTT results like: inadequate sample volumes, heparin contamination, difficult phlebotomy, prolonged pre-analytical storage or high hematocrit, avoiding icteric, hemolytic, lipemic samples (16).

The interpretation of results should take into consideration the great varibility of the sensitivity of reagents used for the aPTT assay to anti-phospholipid antibodies (17); it is also to be underscored that aPTT reagents play an important role, modulating the mixing study depending on their sensitivity to FVIII level (12).

\section{What should the workup list consist of?}

- exploration of LAC by the multiple step procedure with screening, mixing and confirmation steps done by two parallel assays, preferable to add a not fluid-solid phase -ELISA test $(2,18)$.

- comprehensive investigation of hemostasis

Are the platelet count and coagulation factors activities of FXII, XI, X, IX, VIII +/- FII (the latter in rare cases with bleeding phenotype) for their specific inhibitors exploration meaningful? Searching literature for data regarding cases with $\mathrm{AH}$ and concomitant $\mathrm{LAC}$, we have found many reports, some of these describing a therapeutic intervention with either high dose intravenous immunoglobuline (IvIg), corticosteroids, cyclophosphamide or rituximab. In contrast to our patients the majority of the reported cases were clinically characterized by different types of bleeding and received treatment (1924). Critical reviews of such $\mathrm{AH}$ associating LAC considered in frequent cases the diagnosis doubtful $(11,12)$. On the other hand, concerning non-symptomatic patients, it has been shown that about $10 \%$ of $\mathrm{AH}$ are asymptomatic at the time of their first presentation (25). Interestingly, Agostini et al. suggested that the simultaneous presence of LAC might probably mitigate the bleeding episodes and not produce bleeding in AH (21). Moreover, there are reports of patients with asymptomatic onsets who developed later occuring life-threatening bleeds. Ballard et al. reported about a patient with rheumatoid arthritis who's pre-surgical findings were consistent with LAC. At that time of the first presentation the patient was asymptomatic and had no prior history of bleeding. Nevertheless, following a liver biopsy he presented a nearly catastrophic clinical course due to the coexistent AH A which was the cofactor for the initial isolated elevation of aPTT (22). Our answer to this question would be to undertake a comprehensive exploration only in the situation of inaccessibility to more reliable assays. In order to overcome the pitfalls generated by the present diagnostic procedures for LAC, an extension of laboratory assays with chromogenic tests for titration of coagulation factors and ELISA tests for the confirmation of the presence of inhibitors are mandatory (25).

Is it advisable to identify additional risk factors? The isolated transient LAC positivity of asymptomatic persons seems to have an equal risk as in healthy persons, being accepted as an unrisky situation (26). At the same time there are some observations regarding the significantly higher risk of evolution to APS in asymptomatic LAC carriers. Therefore the question remains if we should prolong the follow-up of these persons and if we should extend our exploration in the case of persistent LAC with associated risk factors like obesity, diabetes, malignancy, congenital heart disease, hypercholesterolemia, smoking, oral contraceptive use? Large, multicenter randomised clinical studies will give an evidence-based answer $(27,28)$. 


\section{Who and how should be treated in the case of children with LAC?}

Regarding the therapeutical approach in patients with LAC without presenting any hemorrhagic manifestation, in which the finding of an elevated aPTT is isolated, the attitude remains a subject of debate $(11,12)$. The reasons for applying a "watch and wait" recommendation is the exceptionally low proportion of bleeders, despite of low levels of factors activity $(1,14)$. Immunosupression (steroids, IvIg, plasma exchange) is indicated only in cases with autoimmune background associating LAC and presenting clinical manifestations, whereas in those without suggestive clinical manifestations a thorough workup including ELISA-based assays should be employed prior to proceeding with potentially harmfull immunosuppressive treatment (10-12,22,23).

Concerning surgery in patients with isolated elevated aPTT, in the present context of debate with respect to the opportunity of preoperative routine coagulation screening, we rather follow a prudent attitude of performing routinely aPTT, PT and platelet count prior to surgery or an invasive procedure (16). Nevertheless, in the situation of elevated aPTT associated to a positive asymptomatic LAC we would also perform PCT and eventually global coagulation tests like TEG/TGA (27). In this situation, waiting for the results of a thorough diagnostic workup as presented above, we would recommend the performance without delay of unavoidable surgical interventions as long as appropriate backup hemostatic medication is available. Programmed surgical interventions can be postponed until the diagnosis is completely clarified (9).

\section{Conclusions}

Although rare and mostly innocent considering its clinical impact in asymptomatic children, LAC in children needs to be seriously taken into consideration from the diagnostic point of view in order to avoid extensive and expensive blood workup and sometimes also dangerous therapeutic attitudes. A risk-stratified approach (strenght and persistence of LAC, associated risk factors, aso), usage of new antibody specificities, more sensitive and specific laboratory tests, will bring in the coming years a risk-adapted scoring model and will contribute to the development of new strategies, evaluated for the risk of APS or other manifestations. Further prospective multicenter studies are required to understand clinical outcomes and laboratory correlation in children with positive LAC.

\section{Abbreviations}

aCL - anticardiolipin antibodies aß2GPI - anti-ß2 glycoprotein I antibodies

$\mathrm{AH}$ - acquired haemophilia

aPLs - antiphospholipid antibodies

LAC - lupus anticoagulant

APS - antiphospholipid syndrome

aPTT - activated partial thromboplastin time

BU - Bethesda units

dRVVT - dilute Russel's viper venom time

$\mathrm{F}$ - factor

HSP - Hennoch-Schonlein purpurpura

INR - international normalized ratio

PL - phospholipid

ITP - immune thrombocytopenic purpura

IvIg - intravenous immunoglobuline

LAHS - lupus anticoagulant-hypoprothrombinemia sydrome

PCT - prothrombin consumption test

PT - prothrombin time

SLE - systemic lupus erythematosus

TT - thrombin time

TEG - thrombelastography

TGA - thrombine generation assay

SP - Seidelmeyer purpura 


\section{Conflict of interests}

The authors declare no conflict of interests.

\section{Authors' contribution}

CJ (Conceptualization; Methodology; Formal analysis; Investigation; Resources; Data curation ; Writing - original draft preparation; Writing review)

MS (Conceptualization; Methodology; Formal analysis; Resources; Writing - review and editing; Project administration)

EU (Conceptualization; Writing - original draft preparation; Investigation; Data curation, Visualization)

NAP (Formal analysis; Investigation; Writing original draft preparation)

OB (Formal analysis; Investigation; Writing original draft preparation)

DS (Formal analysis; Methodology; Writing original draft preparation)

ML (Formal analysis; Methodology; Writing original draft preparation)

AM (Resources; Formal analysis; Writing original draft preparation; Visualization)

AT (Writing draft; Writing - critical review; Conceptualization)

SA (Conceptualization; Methodology; Supervision; Writing - review and editing;)

\section{References}

1. Vivaldi P, Rossetti G, Galli M, Finazzi G. Severe bleeeding due to acquired hypoprothrombinemia-lupus anticoagulant syndrome. Case report and review of literature. Haematologica. 1997;82:345-7.

2. Pengo V. ISTH guidelines on Lupus Anticoagulant testing. Throm Res. 2012;130:S76-7. DOI: 10.1016/j. thromres.2012.08.283

3. Male C, Lechner K, Eichinger S, Kyrle PA, Kapiotis S, Wank H, et al. Clinical significance of lupus anticoagulants in children. J Pediatrics. 1999;134:199-205. DOI: 10.1016/S0022-3476(99)70416-6

4. Knobe K, Tedgård U, Ek T, Sandström PE, Hillarp A. Lupus anticoagulants in two children-bleeding due to nonphospholipid-dependent antiprothrombin anti- bodies. Eur J Pediatr. 2012 Sep;171(9):1383-7. DOI: 10.1007/s00431-012-1737-1

5. Gómez-Puerta JA, Cervera R. Diagnosis and classification of the antiphospholipid syndrome. J Autoimmun. Feb-Mar 2014;48-49:20-5. DOI: 10.1016/j. jaut.2014.01.006

6. Desposito F, Arkel Y. Inhibitors of coagulation in children. Crit Rev Oncol Hematol. 1987;7(1):53-69. DOI: 10.1016/S1040-8428(87)80014-8

7. Moore GW. Current Controversies in Lupus Anticoagulant Detection. Antibodies (Basel). 2016 Dec 2;5(4):22. DOI: 10.3390/antib5040022

8. Ames PRJ, Graf M, Archer J, Scarpato N, Iannaccone L. Prolonged activated partial thromboplastin time: Difficulties in discriminating coexistent factor VIII inhibitor and lupus anticoagulant. Clin Appl Thromb Hemost. 2015 Mar;21(2):149-54. DOI: $10.1177 / 1076029614541516$

9. Kallanagowdar C, Chauhan A, Puertolas MV, Warrier R. Prevalence and Resolution of Lupus Anticoagulant in Children. Ochsner J. 2016;16(2):172-5.

10. Swadzba J, Iwaniec T, Pulka M, de Laat B, de Groot PG, Musial J. Lupus anticoagulant: Performance of the tests as recommended by the latest ISTH guidelines. J Thromb Haemost. 2011 Sep;9(9):1776-83. DOI: 10.1111/j.1538-7836.2011.04420.x

11. Ames PRJ, Montero D, Archer J. Co-existent Acquired Haemophilia and Lupus Anticoagulant. A Thorny Issue. Indian J Hematol Blood Transfus. 2016 Jun; 32(Suppl 1): 248. DOI: $10.1007 / \mathrm{s} 12288-015-0572-8$

12. Penmetsa GK, Rodgers GM, Smock KJ. Avoiding errors in the laboratory evaluation of potent lupus anticoagulants. Am J Hematol. 2010 Apr;85(4):272-4. DOI: 10.1002/ajh.21666

13. Wincup C, Ioannou Y. The differences between childhood and adult onset antiphospholipid syndrome. Front Pediatr. 2018 Nov 27;6:362. DOI: 10.3389/ fped.2018.00362

14. Malbora B, Bilaloglu E. Lupus anticoagulant positivity in pediatric patients with prolonged activated partial thromboplastin time: A single-center experience and review of literature. Pediatr Hematol Oncol. 2015;32(7):495-504. DOI: 10.3109/08880018.2015.1065302

15. Aguiar CL, Soybilgic A, Avcin T, Myones BL. Pediatric Antiphospholipid Syndrome. Curr Rheum Rep. 2015;17. DOI: 10.1007/s11926-015-0504-5

16. Tagariello G, Radossi P, Salviato R, Zardo M, de Valentin L, Basso M, et al. Clinical relevance of isolated prolongation of the activated partial thromboplastin time in a cohort of adults undergoing surgical procedures. Blood Transf. 2017;15:557-61.

17. Li R, Swaelens C, Vandermijnsbrugge F, Cantinieaux B. Applying a direct aPTT ratio (PlatelinLS/ActinFS) permits to identify rapidly and reliably a bleeding-related 
factor deficiency or a lupus anticoagulant sequential to an isolated prolongation of aPTT in paediatric pre-operative screening. Euro J Haematol. 2016;96:578-85. DOI: $10.1111 /$ ejh. 12634

18. Devreese KMJ, Ortel TL, Pengo V, de Laat B. Laboratory criteria for antiphospholipid syndrome: communication from the SSC of the ISTH. J Thromb Haemost. 2018;16:809-13. DOI: 10.1111/jth.13976

19. Dreisbach JD, Dreisbach LP, Young DE, Dreisbach PB. Acquired factor VIII inhibitor and lupus anticoagulant presenting with prolonged aPTT: a case report. Grand Rounds. 2010;10:19-24.

20. Brings HA, Waas JK, McCrae KR, Baele HR, Goldstone J. Successful management of life-threatening hemorrhage in a patient with synchronous lupus anticoagulant and factor VIII inhibitor. J Vasc Surg. 2002 Oct;36(4):853-5. DOI: 10.1067/mva.2002.127336

21. Agostini V, Biasoli C, Biguzzi R, Santarelli R, Leoni P. Case Report: Coexistence of Factor VIII Inhibitor and Lupus Anticoagulant. Blood. 2007;110:3948. DOI: 10.1182/blood.V110.11.3948.3948

22. Ballard HS, Nyamuswa G. Life-threatening haemorrhage in a patient with rheumatoid arthritis and a lupus anticoagulant coexisting with acquired autoantibodies against factor VIII. Br J Rheumatol. 1993 Jun;32(6):515-7. DOI: 10.1093/rheumatology/32.6.515

23. Seethala S, Collins NP, Comerci G. An Unusual Etiology for Elevation of Activated Partial Thromboplastin
Time (aPTT) in SLE: Acquired Hemophilia and Lupus Anticoagulant. Case Rep Hematol 2013;2013:1-3. DOI: $10.1155 / 2013 / 521785$

24. Taher A, Abiad R, Uthman I. Coexistence of lupus anticoagulant and acquired haemophilia in a patient with monoclonal gammopathy of unknown significance. Lupus. 2003;12:854-6. DOI: 10.1191/0961203303lu463cr

25. Tiede A, Werwitzke S, Scharf RE. Laboratory Diagnosis of Acquired Hemophilia A: Limitations, Consequences, and Challenges. Semin Thromb Hemost. 2014 Oct;40(7):803-11. DOI: 10.1055/s-0034-1390004

26. Mustonen $\mathrm{P}$, Lehtonen KV, Javela K, Puurunen M. Persistent antiphospholipid antibody (aPL) in asymptomatic carriers as a risk factor for future thrombotic events: a nationwide prospective study. Lupus. 2014 Dec;23(14):1468-76. DOI: $10.1177 / 0961203314545410$

27. Sciascia S, Baldovino S, Schreiber K, Solfietti L, Radin M, Cuadrado MJ, et al. Thrombotic risk assessment in antiphospholipid syndrome: The role of new antibody specificities and thrombin generation assay. Clin Mol Allergy. 2016; 14:6. DOI: 10.1186/s12948-016-0043-2

28. Radin M, Schreiber K, Costanzo P, Cecchi I, Roccatello D, Baldovino S, et al. The adjusted Global AntiphosPholipid Syndrome Score (aGAPSS) for risk stratification in young APS patients with acute myocardial infarction. Int J Cardiol. 2017 Aug 1;240:72-77. DOI: 10.1016/j.ijcard.2017.02.155 